\title{
INDUCED ACYCLIC TOURNAMENTS IN RANDOM DIGRAPHS: SHARP CONCENTRATION, THRESHOLDS AND ALGORITHMS ${ }^{1}$
}

\author{
Kunal Dutta and C.R. Subramanian \\ The Institute of Mathematical Sciences \\ Taramani, Chennai-600113, India \\ e-mail: kdutta@imsc.res.in \\ crs@imsc.res.in
}

\begin{abstract}
Given a simple directed graph $D=(V, A)$, let the size of the largest induced acyclic tournament be denoted by $\operatorname{mat}(D)$. Let $D \in \mathcal{D}(n, p)$ (with $p=p(n))$ be a random instance, obtained by randomly orienting each edge of a random graph drawn from $\mathcal{G}(n, 2 p)$. We show that mat $(D)$ is asymptotically almost surely (a.a.s.) one of only 2 possible values, namely either $b^{*}$ or $b^{*}+1$, where $b^{*}=\left\lfloor 2\left(\log _{r} n\right)+0.5\right\rfloor$ and $r=p^{-1}$.

It is also shown that if, asymptotically, $2\left(\log _{r} n\right)+1$ is not within a distance of $w(n) /(\ln n)$ (for any sufficiently slow $w(n) \rightarrow \infty)$ from an integer, then $\operatorname{mat}(D)$ is $\left\lfloor 2\left(\log _{r} n\right)+1\right\rfloor$ a.a.s. As a consequence, it is shown that $\operatorname{mat}(D)$ is 1-point concentrated for all $n$ belonging to a subset of positive integers of density 1 if $p$ is independent of $n$. It is also shown that there are functions $p=p(n)$ for which $\operatorname{mat}(D)$ is provably not concentrated in a single value. We also establish thresholds (on $p$ ) for the existence of induced acyclic tournaments of size $i$ which are sharp for $i=i(n) \rightarrow \infty$.

We also analyze a polynomial time heuristic and show that it produces a solution whose size is at least $\log _{r} n+\Theta\left(\sqrt{\log _{r} n}\right)$. Our results are valid as long as $p \geq 1 / n$. All of these results also carry over (with some slight changes) to a related model which allows 2-cycles.
\end{abstract}

Keywords: random digraphs, tournaments, concentration, thresholds, algorithms.

2010 Mathematics Subject Classification: 05C80.

\section{REFERENCES}

\footnotetext{
${ }^{1}$ A preliminary version of parts of this work appeared as an extended abstract in LATIN, 2010, Oaxaca, Mexico.
} 
[1] D. Achlioptas and A. Naor, The two possible values of the chromatic number of a random graph, Ann. of Math. 162 (2005) 1333-1349.

doi:10.4007/annals.2005.162.1335

[2] N. Alon and M. Krivelevich, The concentration of the chromatic number of random graphs, Combinatorica 17 (1997) 303-313. doi:10.1007/BF01215914

[3] N. Alon and J.H. Spencer, The Probabilistic Method (Wiley International, 2001).

[4] B. Bollobás, Random Graphs (2nd Edition, Cambdrige University Press, 2001). doi:10.1017/CBO9780511814068

[5] B. Bollobás and P. Erdős, Cliques in random graphs, Math. Proc. Cambridge Philos. Soc. 80 (1988) 419-427. doi:10.1017/S0305004100053056

[6] B.K. Rosen, Robust linear algorithms for cutsets, J. Algorithms 3 (1982) 205-217. doi:10.1016/0196-6774(82)90020-7

[7] K. Dutta and C.R. Subramanian, Largest induced acyclic tournament in random digraphs: A 2-point concentration, in: Proceedings of LATIN-2010 (9th Latin American Theoretical Informatics Symposium), Oaxaca, Mexico, April 2010.

[8] K. Dutta and C.R. Subramanian, Induced acyclic subgraphs in random digraphs: Improved bounds, in: Proceedings of AofA-2010 (21st Internatioanl Meeting on Probabilistic and Asymptotic Methods for the Analysis of Algorithms), Vienna, Austria, June 2010, to appear.

[9] R.W. Floyd Assigning meaning to programs, Proc. Sympos. Appl. Math. 19 (1967) 19-32.

[10] A.M. Frieze, On the independence number of random graphs, Discrete Math. 81 (1990) 171-176. doi:10.1016/0012-365X(90)90149-C

[11] M.R. Garey and D.S. Johnson, Computers and Intractability: A Guide To The Theory of NP-Completeness (W.H.Freeman, San Francisco, 1978).

[12] J. Hastad, Clique is hard to approximate within $n^{1-\varepsilon}$, Acta Math. 182 (1999) 105142 . doi:10.1007/BF02392825

[13] S. Janson, T. Łuczak and A. Ruciński, Random Graphs (John Wiley \& Sons, Inc., 2000). doi:10.1002/9781118032718

[14] S. Khot, Improved inapproximability results for maxclique, chromatic number and approximate graph coloring, in: Proceedings of the 42nd IEEE Symp. Foundations of Computer Science (FOCS 2001) 600-609.

[15] M. Krivelevich and B. Sudakov, Coloring random graph, Inform. Process. Lett. 67 (1998) $71-74$

doi:10.1016/S0020-0190(98)00092-1 
[16] T. Łuczak, A note on the sharp concentration of the chromatic number of random graphs, Combinatorica 11 (1991) 295-297. doi: $10.1007 / \mathrm{BF} 01205080$

[17] C. Lund and M. Yannakakis, The approximation of maximum subgraph problems, Proceedings of the 20th International Colloquium on Automata, Languages and Programming (ICALP'93), Lecture Notes in Comput. Sci. 700 (1993) 40-51.

[18] M. Cai, X. Deng and W. Zang, An approximation algorithm for feedback vertex set in tournaments SIAM J. Comput. 30 (2001) 1993-2007. doi:10.1137/S0097539798338163

[19] R. Motwani and P. Raghavan, Randomized Algorithms (Cambridge University Press, 1995). doi:10.1017/CBO9780511814075

[20] C.H. Papadimitriou and M. Yannakakis Optimization, approximation, and complexity classes, J. Comput. System Sci. (Special issue for the 20th ACM Symposium on Theory of Computing) 43 (1991) 425-440.

[21] E. Speckenmeyer, On feedback problems in digraphs, Proceedings of the 15th International Workshop on Graph Theoretic Concepts in Computer Science (WG'89), Springer-Verlag, Lecture Notes in Comput. Sci. 411 (1990) 218-231. doi:10.1007/3-540-52292-1_16

[22] J.H. Spencer and C.R. Subramanian, On the size of induced acyclic subgraphs in random digraphs, Discrete Math. Theor. Comput. Sci. 10 (2008) 47-54.

[23] C.R. Subramanian, Finding induced acyclic subgraphs in random digraphs, Electron. J. Combin. 10 (2003) \#R46.

\section{APPEndiX}

\section{1. $\operatorname{mat}(D)$ versus $\omega(G)$}

The following lemma relates the probabilities in the two models $\mathcal{D}(n, p)$ and $\mathcal{G}(n, p)$ for having, respectively, tournaments and cliques of specific sizes. Its proof is similar to the proof of an analogous relationship involving mas $(D)$ and $\alpha(G)$ (maximum size of an independent set in $G$ ) established in [23].

Lemma 1.1. For any positive integer $b$, for a random digraph $D \in \mathcal{D}(n, p)$,

$$
\operatorname{Pr}[\operatorname{mat}(D) \geq b] \geq \operatorname{Pr}[\omega(G) \geq b],
$$

where $G \in \mathcal{G}(n, p)$.

Proof. Given a linear ordering $\sigma$ of vertices of $D$ and a subset $A$ of size $b$, we say that $D[A]$ is consistent with $\sigma$ if for every $\sigma_{i}, \sigma_{j} \in A$ with $i<j, D[A]$ has the $\operatorname{arc}\left(\sigma_{i}, \sigma_{j}\right)$. 
Let $\tau$ denote an arbitrary but fixed ordering of $V$. Once we fix $\tau$, the spanning subgraph of $D$ formed by arcs of the form $(\tau(i), \tau(j))(i<j)$ is having the same distribution as $\mathcal{G}(n, p)$. Hence, for any $A$, the event of $D[A]$ being consistent with $\tau$ is equivalent to the event of $A$ inducing a clique in $\mathcal{G}(n, p)$. Hence,

$$
\begin{aligned}
\operatorname{Pr}(\text { mat }(D) \geq b) & =\operatorname{Pr}(\exists A,|A|=b, D[A] \text { is an acyclic tournament }) \\
& =\operatorname{Pr}(\exists A,|A|=b, \exists \sigma, D[A] \text { is consistent with } \sigma) \\
& =\operatorname{Pr}(\exists \sigma, \exists A,|A|=b, D[A] \text { is consistent with } \sigma) \\
& \geq \operatorname{Pr}(\exists A,|A|=b, D[A] \text { is consistent with } \tau) \\
& =\operatorname{Pr}(\omega(G) \geq b) .
\end{aligned}
$$

Hence it is natural that we have a bigger upper bound for mat $(D)$ than we have for $\omega(G)$.

Note: Recall that we first draw an undirected $G \in \mathcal{G}(n, 2 p)$ and then choose uniformly randomly an orientation of $E(G)$. Hence, for any fixed $A \subseteq V$ of size $b$ with $b=\omega(1)$,

$$
\operatorname{Pr}(D[A] \text { is an acyclic tournament } \mid G[A] \text { induces a clique })=\frac{b !}{2^{\left(\begin{array}{c}
b \\
2
\end{array}\right)}}=o(1)
$$

However, there are so many cliques of size $b$ in $G$ that one of them manages to induce an acyclic tournament.

\subsection{Proof of Theorem ??}

We reduce the NP-complete Maximum Clique problem $\operatorname{MC}(G, k)$ to the $\operatorname{MAT}(D$, $k)$ problem as follows. Given an instance $(G=(V, E), k)$ of the first problem, compute an instance $f(G)=\left(G^{\prime}=(V, A), k\right)$ in polynomial time where

$$
A=\{(u, v): u v \in E, u<v\}
$$

Clearly, $G^{\prime}$ is a dag and it is easy to see that a set $V^{\prime} \subseteq V$ induces a clique in $G$ if and only if $V^{\prime}$ induces an acyclic tournament in $G^{\prime}$. This establishes that $\operatorname{MAT}(D, k)$ is NP-hard even if $D$ is restricted to be a dag.

The inapproximability of $\operatorname{MAT}(D)$ follows from the following observation. Note that the reduction $G \rightarrow f(G)$ is an $L$-reduction in the sense of [20], since $|f(G)|=|G|$ and $\omega(G)=\operatorname{mat}\left(G^{\prime}\right)$. Hence, any inapproximability result on maximum clique in undirected graphs (for example $[12,14]$ ), implies a similar inapproximability for the $\operatorname{MAT}(D)$ problem. 


\subsection{Proof of Claim ??}

Order the vertices of $U$ along a Hamilton path $P$ (if any exists) of $H$. An arc $(u, v) \in A$ is a forward arc if $u$ comes before $v$ in $P$ and is a backward arc otherwise. Since $H$ is acyclic, any $\operatorname{arc}(v, u) \in A$ must be a forward arc, since otherwise the segment of $P$ from $u$ to $v$ along with $(v, u)$ forms a cycle in $H$.

Now if there is another Hamilton path $Q$ in $H, Q \neq P$, then walking along $P$, consider the first vertex $a$ where $Q$ differs from $P$. Then in the path $Q, a$ is visited immediately after some vertex $a^{\prime}$ that comes after $a$ in $P$. But this implies that $\left(a^{\prime}, a\right)$ is a backward arc in $H$ contradicting the observation earlier that $H$ has no backward arc.

\subsection{Remaining cases of Theorem ??}

For $1 / w n \leq p<1 / n$,

$$
E[X(n, 4)]=\left(\begin{array}{l}
n \\
4
\end{array}\right) \cdot 4 ! \cdot p^{\left(\begin{array}{l}
4 \\
2
\end{array}\right)} \leq n^{4} p^{6} \leq\left(1 / n^{2}\right)=o(1) .
$$

Now, an acyclic tournament of size 2 is simply an edge which a.a.s. exists since:

$\operatorname{Pr}[\operatorname{mat}(D)<2]=\operatorname{Pr}[D$ is the empty graph $]=(1-2 p)^{\left(\begin{array}{l}n \\ 2\end{array}\right)} \leq e^{-n(n-1) p}=o(1)$,

since $p \geq 1 / w n \geq w / n^{2}$. Hence, when $1 / w n \leq p \leq 1 / n$, $\operatorname{mat}(D) \in\{2,3\}$, a.a.s.

For $w n^{-2} \leq p<1 / w n$,

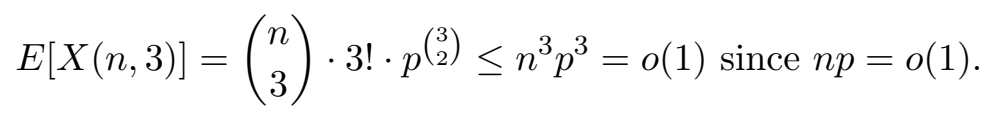

The proof for $\operatorname{mat}(D) \geq 2$ is the same as in the previous case, since $n^{2} p=$ $\omega(1)$, and hence, at least one arc will exist, a.a.s. So when $w / n^{2} \leq p \leq 1 / w n$, $\operatorname{mat}(D)=2$, a.a.s.

For $\left(w n^{2}\right)^{-1} \leq p \leq w / n^{2}, E[X(n, 3)]=o(1)$, as in the previous case, and so $\operatorname{mat}(D)=1$ or 2 , a.a.s. When $p<\left(w n^{2}\right)^{-1}, \operatorname{mat}(D)=1$ since $D$ a.a.s. has no directed edge. 